\title{
Ultrastructural characteristics of ostrich eggshell: outer shell membrane and the calcified layers
}

\author{
P D G Richards ${ }^{a}$, P A Richards ${ }^{a}$ and M E Lee ${ }^{b}$
}

\begin{abstract}
The ultrastructure of the eggshell of the domestic hen has been well researched and structural studies of other avian species, such as the ostrich, often basetheir interpretation of egg shell structure on that of the chicken. In the ostrich, lowered hatchability and hatching trauma may be due to shell ultrastructural abnormalities. In the present study the ultrastructure of the calcified portion, and the outer shell membrane (OSM), of domesticated ostrich eggshells was investigated using standard electron microscopic techniques. Transmission and scanning electron microscopy studies demonstrated intimatecontact between cup-shaped structurespresent on theOSM and themammillary layer of thecalcified portion of the shell. The initial calcium carbonate growth of the calcified shell was of a dendritic nature with nucleation sites on the surface of the cup's contents. The dendritic growth gave way to a more randomly-orientated, smaller crystallite growth structure, which changed in form as it neared the vertical crystal layer (VCL). TheVCL is described as being both amorphous and 'crumbly' depending on the plane of fracture. These observations suggest that firstly, initial calcification is contained within the cups and is then directed outwards to form the shell and that secondly, the VCL may contain an evolutionary, calcified cuticular layer. These observations serve as a baselinefor studies investigating the effect of shell structureand strength on hatchling trauma and theinfluence of maternal diet.
\end{abstract}

Key words: biomineralisation, calcification, EDS, electron microscopy, shell membrane.

Richards P D G, Richards P A, Lee M E Ultrastructural characteristics of ostrich eggshell: outer shell membrane and the calcified layers. J ournal of theSouth A frican Veterinary A ssociation 71(2): 97-102 (En.). Department of Anatomy, Faculty of Medicine, University of Pretoria, PO Box 2034, Pretoria, 0001 South Africa.

\section{INTRODUCTION}

The ultrastructure of the eggshell of the domestic hen (Gallus gallus domesticus) is well described ${ }^{6,17,30}$. As commercial interests are invested in egg quality, the relationship of the structure of the shell and egg quality has also been investigated using various microscopy techniques ${ }^{6,9}$. Such studies have used fracture techniques to demonstrate the presence of between 5 and 6 layers associated with theeggshell, which tend to beremarkably constant across most avian species. The typical identifiable layers of the eggshell (Fig. 1), from the inside to the outside of the shell, are the: inner shell membrane, outer shell membrane (OSM), mammillary layer, palisade layer, vertical crystal layer (VCL) and the cuticle/shell accessory material.

Most authorities are in agreement that,

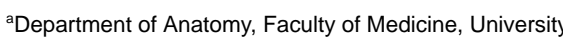
of Pretoria, Pretoria, PO Box 2034, Pretoria, 0001 South Africa. E-mail: richards@medic.up.ac.za

${ }^{b}$ Electron Microscope Unit, Faculty of Mathematics and Natural Science, University of the North, Sovenga, South Africa.

Received: November 1999. Accepted: April 2000.
Calcium carbonate accumulates around these nucleation sites, forming the calci- in thecase of theostrich (Struthio camelus), ent is thought to start in organic nodules that are randomly distributed on the outer shell membrane ${ }^{27}$. fied portion of the egg, which is then coated with theorganic layer beforebeing laid $^{12}$. The ultimate strength of the eggshell of thechicken is thought to be determined by both the bird's calcium ration during the initial hours of calcification and by the number of nucleation sites present on the outer shell membrane ${ }^{12}$. The nucleation sites are probably genetically controlled and are thus not manipulableby the farmer ${ }^{12}$. It is unlikely that the architecture of the shell's outer membrane playsany decisive rolein theinherent strength of the shell ${ }^{12}$.

Most research on the eggshell of the ostrich has tended to concentrate on the calcified components of the shell (mammillary layer, palisade and vertical crystal layer) ${ }^{11,23,25}$. Sparks and Deeming ${ }^{23}$ used the fracture method to demonstrate that the features of the eggshell of $\mathrm{S}$. camelus are similar to those of the domestic chicken. Qualitative observations have suggested that there is less porosity in the palisade layer of these eggshells compared to those of the chicken and other avian species ${ }^{5,13}$. Thus a highly porous eggshell, such as that of a domestic chicken, is likely to have a higher fragility index and therefore lower breaking strength compared to an eggshell that is less porous. Therehas been little research on the interface relationship between the calcified portions of the shell and the OSM, as this is assumed to be similar to that found in the chicken ${ }^{23}$. This interface is a determinant of shell strength in the domestic chicken ${ }^{12}$ and may play a similar

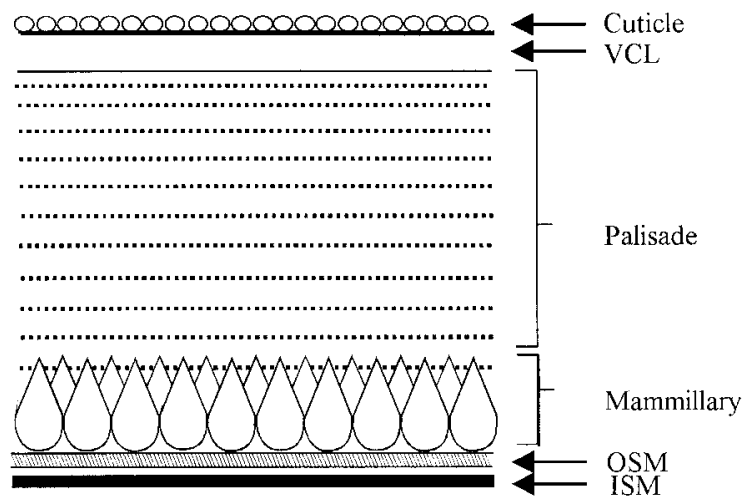

Fig. 1: Schematic representation of the eggshell of avian species. ISM = inner shell membrane, OSM = outer shell membrane, $\mathrm{VCL}=$ vertical crystal layer. 
role in the ostrich egg. Deeming ${ }^{14}$ has shown that theostrich, unlike other avian species, hatches with the assistance of its limbs, as it has no egg tooth. Hence, any increase in eggshell strength as a result of breeding or diet may result in trauma to the 'hatching' leg. On ostrich farms, in both Australia and southern Africa, over $20 \%$ of chicks suffer trauma to their right limb, the 'hatching' leg, during the hatching process $^{14}$. A more comprehensive picture of the ostrich eggshell is required to determine the structural basis for eggshell strength in this species in order to reducetheincidence of hatching trauma.

In the present study, shells of normal fertile ostrich eggs (S. c. australis var. domesticus) were examined to determine their ultrastructure in relation to previous descriptions $s^{16,23,24,26,28}$. Furthermore, the relationship between the mammillary layer and the OSM was investigated as, firstly, this intimate contact may have a bearing on ultimate hatchability and secondly, the relationship has not been fully described for thisspecies, although it is usually assumed to correspond to that of the chicken egg.

\section{MATERIALS AND METHODS}

Ostrich eggshells, from recently hatched chicks, were collected from farms in the vicinity of Onderstepoort, Gauteng, South Africa. The incubation and hatching of these eggs was normal and no hatching trauma or pathology was observed in the young birds.

\section{Outer shell membrane}

Fragments of the outer shell membrane (OSM) were removed from theshells and processed for scanning electron microscopy (SEM), energy dispersive X-ray analysis (EDS) and transmission electron microscopy (TEM) using the techniques described below. Fragments of OSM were mounted on aluminium stubs, for SEM and EDS, so that the outer surface (that normally attached to the mammillary layer) faced the detector. The samples for SEM were then gold sputter-coated and viewed using a JEOL ${ }^{\circledR} 840$ SEM with an accelerating voltage of $5 \mathrm{kV}$. The EDS samples were viewed, uncoated, using a JEOL ${ }^{\circledR} 5800 L V$ SEM with an accelerating voltage of $25 \mathrm{kV}$. The spectra were obtained under low-vacuum conditions (0.3 torr) with a Noran Voyager ${ }^{\circledR}$ fully quantitative EDS system. The fragments of OSM, prepared for TEM, were fixed in $2.5 \%$ glutaraldehydein phosphatebuffer (pH 7.2) for 60 minutes and, following a buffer wash, were post-fixed in $1 \%$ osmium tetroxide for 30 minutes at room temperature. The samples were processed and embedded in LR White ${ }^{\circledR}$ resin
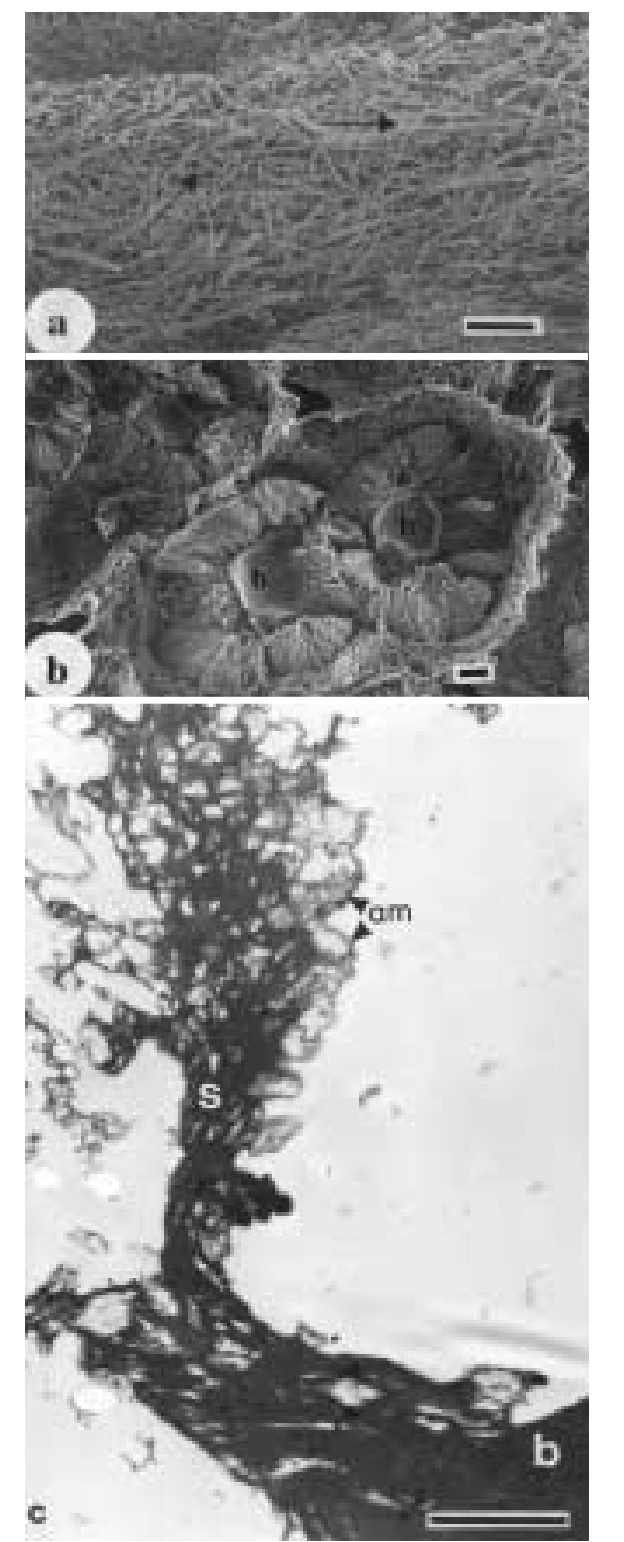

Fig. 2: Electron micrographs of the outer shell membrane (OSM): a) SEM micrograph of an oblique section through the OSM. Arrows indicate the differing orientation of the fibres of 2 layers (scale bar $=50 \mu \mathrm{m}$ ); b) SEM micrograph of the OSM, viewed from the direction of the calcified shell, showing the cup-shaped structures on the upper surface. The amorphous mass (b) is the basal area of the cup (scale bar $=10 \mu \mathrm{m}$ ); c) TEM micrograph of the wall of a cup-shaped structure. $\mathrm{s}=$ side wall, $\mathrm{b}=$ basal area, $\mathrm{am}=$ amorphous material (scale bar $=1 \mu \mathrm{m}$ ).

using standard methods. Thin sections were obtained from the resultant blocks and stained with uranyl acetate and lead citrate before examination with aPhilips ${ }^{\circledR}$ 301 TEM using an accelerating voltage of $80 \mathrm{kV}$.

\section{Calcified shell}

Shell fragments from 6 different shells were examined using the SEM. To maximise examination, the fragments were either radially fractured (with or without the OSM attached), prepared by the etching and polishing technique of
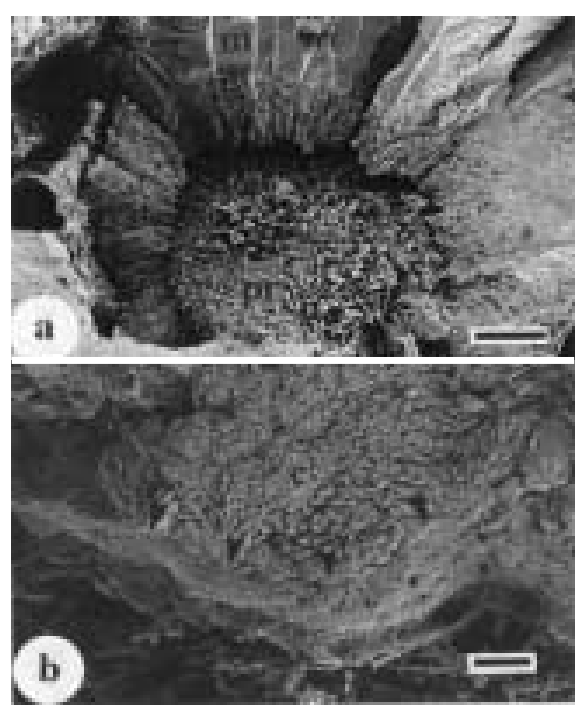

Fig. 3: Scanning electron micrographs of the interface between the OSM and mammillary layer (m): a) the more openly arranged plate-/rod-like crystallites situated within the cup (pl) (scale bar $=20 \mu \mathrm{m}) ; \mathrm{b})$ fractured shell with OSM attached. $c=$ cup contents, arrowheads denote inner surface of the cup. The mammillary layer extends above the area of the micrograph (scale bar $=10 \mu \mathrm{m}$ ).

Richards et al. ${ }^{20}$ or by deep-etching in $8 \%$ EDTA and $6 \%$ formal dehyde for 48 days. The specimens so obtained were mounted on aluminium stubs in such a manner as to present either 1 ) the radial section (fractured or etched), 2) the mammillary layer/OSM interface or 3) theinner aspect of the shell (mammillary layer/OSM surface) towards the detector. All specimens were gold sputter-coated and examined using a $\mathrm{JEOL}^{\circledR} 840$ scanning electron microscope with an accelerating voltage of $5 \mathrm{kV}$.

\section{RESULTS}

\section{Outer shell membrane}

The OSM is composed of a number of layers of fibrous material. The fibres in each layer appear to run at right angles to the layer above and below (Fig. 2a). The upper fibrous layer, that closest to the mammillary layer, has numerous cupshaped structures on its upper surface that face the mammillary layer (Fig. 2b). These structures appear to be formed from the same material as that which forms the underlying OSM. The basal area of the cup appears to be a thickened denselayer (Fig. 2b,c) with numerousoutgrowths that curve around to form the sides of the cup. When examined with TEM, the cup sides have an extremely complex structure, with areas of lightly staining amorphous material forming part of the inner surfaces (Fig. 2c). The inner surface of the cup wall is closely associated with a region of irregularly calcified material located just deep to the 


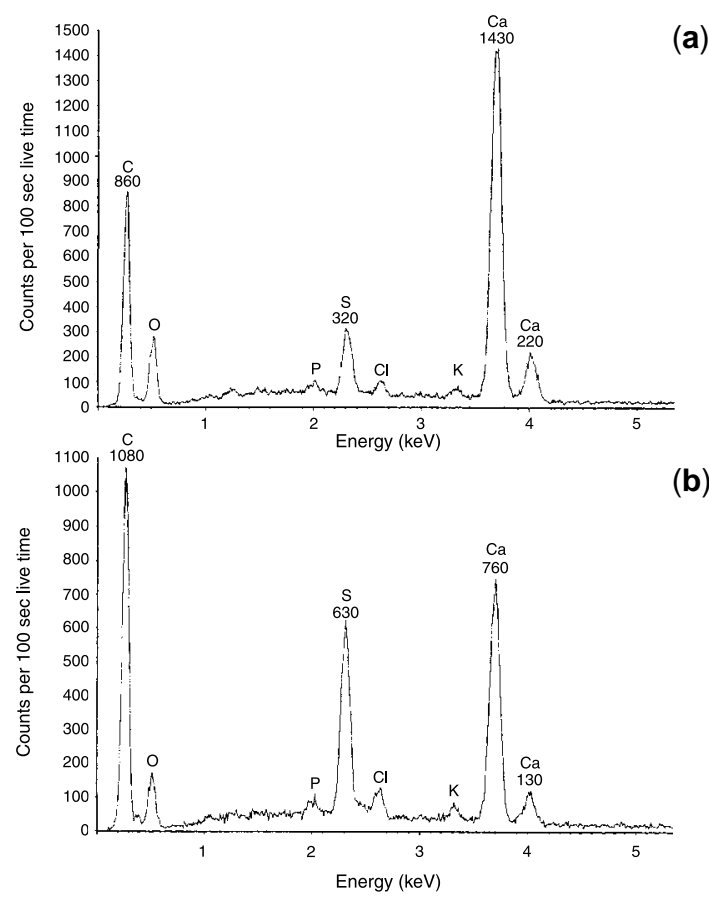

Fig. 4: Energy dispersive spectrum of (a) the cup-shaped structures and (b) the fibrous portion of the OSM. Each major peak is identified and the count for the carbon (C), sulphur (S) and calcium $(\mathrm{Ca})$ peaks is given. Note the difference between the carbon, sulphur and calcium peaks in the 2 areas.

mammillary layer and forming the cup contents. In the fractured radial sections, wheretheOSM was present, theinterface between thecup and this region of irregular calcification could be examined (Fig. 3). The contents of the cup display a more open structure, when compared to the mammillary layer located immediately above, with the crystallites arranged in a plate/rod-like configuration (Fig. 3a). The amorphous protrusions of the inner cup wall, which are clearly seen in thesectioned material, are not in evidence in the SEM preparations, although thereis close contact between thecup contents and the walls of the cup (Fig. 3b). Analysis, using EDS, of the fibrous portion of the OSM and the cup-like structures shows differ-

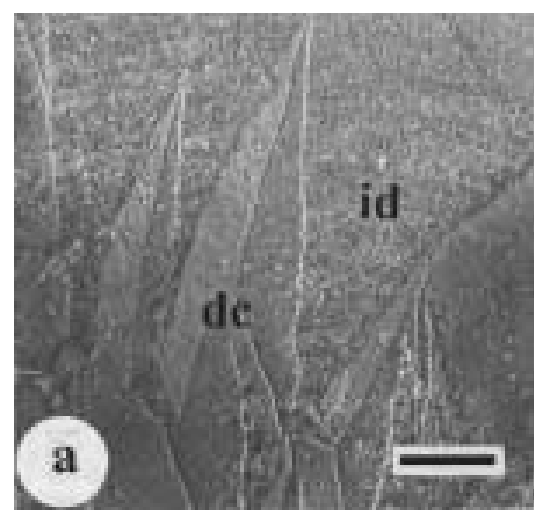

ences in elemental composition between the 2 areas (Fig, 4; Table 1). The cup-like structures have a higher calcium content than the fibrous portion of the OSM, while the sulphur and carbon content of thefibrous part of the OSM isgreater than that of the cups.

\section{Calcified shell}

Scanning electron microscopy of the mammillary layer of the etched ostrich eggshell demonstratesa dendritic growth pattern for the calcium carbonate crystallites in this region (Fig. 5a). The dendritic growth appears to be initiated at nucleation points on the upper surface of the OSM cup contents (Fig. 5b). The basal width of the dendritic 'columns' varies

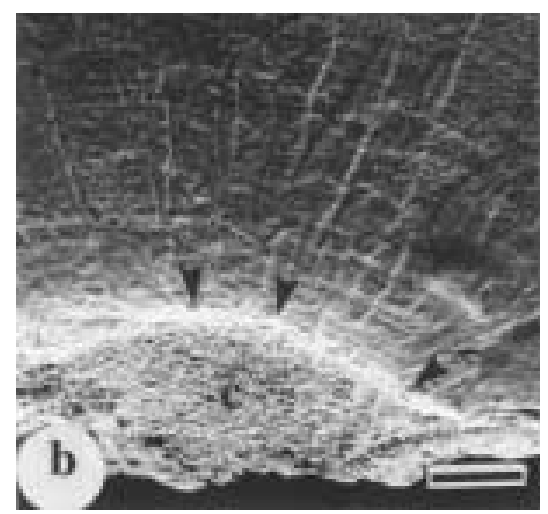

Fig. 5: Scanning electron micrographs of the mammillary layer: a) sample etched using the technique of Richards et al ${ }^{20}$ showing the dendritic crystallites (dc) and inter-dendritic growth area (id) (scale bar $=20 \mu \mathrm{m})$; b) etched sample showing the probable nucleation sites (arrowheads) of the dendritic growth of the mammillary layer on the surface of the cup contents, (c) the remainder of the cup and OSM have been removed from the specimen (scale bar $=20 \mu \mathrm{m}$ ).

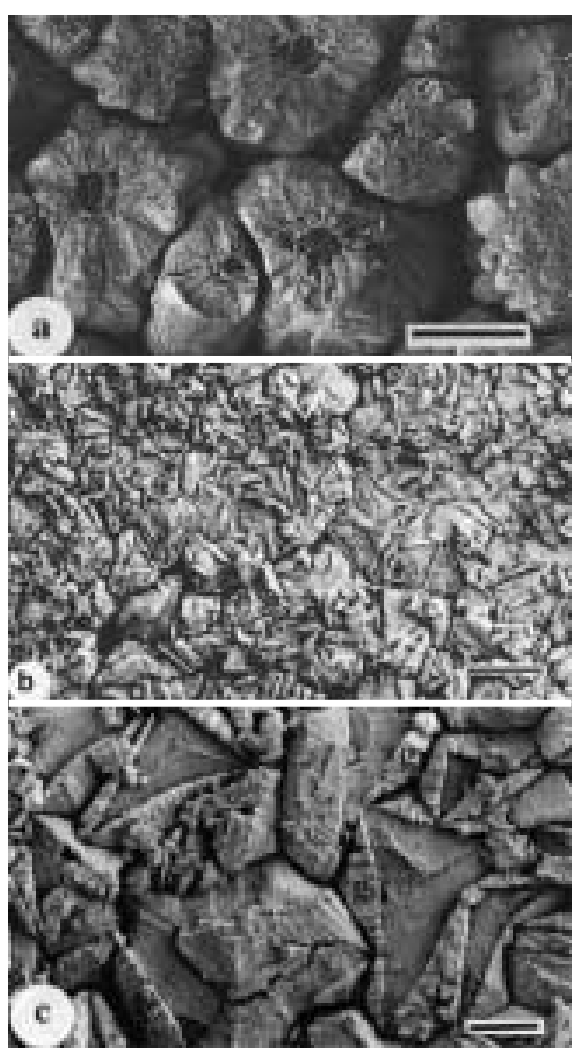

Fig. 6: Scanning electron micrographs of the basal area of the mammillary layer observed from the inner aspect. a) before deep etching (scale bar $=100 \mu \mathrm{m}) ; \mathrm{b}$ ) and $\mathrm{c}$ ) after deep-etching (scale bars $=100 \mu \mathrm{m}$ and $20 \mu \mathrm{m}$, respectively). Note the domed structures before deep-etching and the packed crystallite structures (delineated areas) with differing grains $(\mathrm{g})$ post-etching.

between 90 and $150 \mu \mathrm{m}$ in diameter. A crystallite growth pattern, with a greater degree of randomness, is observed between the dendritic growth patterns and this extends upwards and amalgamates with the crystallites of the palisade layer. This inter-dendritic growth region contains numerous micropores. The micropores are of 2 different sizes, the larger being $1 \mu \mathrm{m}$ in diameter and the smaller $0.3 \mu \mathrm{m}$ in diameter. The larger pores are distributed in a horizontal banding pattern, while the smaller pores have a more random distribution between the bands of the larger pores. The dendritic crystallites extend approximately $500 \mu \mathrm{m}$ from the nucleation point outwards into the palisade layer. The inner surface of the shell, with the OSM removed, shows numerous domed features that consist of successive plate or fused rod crystallite growth structures, which have a more open pattern compared to the dendritic growth structures of the mammillary layer (Fig. 6a). Deepetching removes the domed structures and the revealed surface demonstrates a pattern of closely packed crystallites (Fig. 6b). A number of different grains are present within each delineated crystallite 


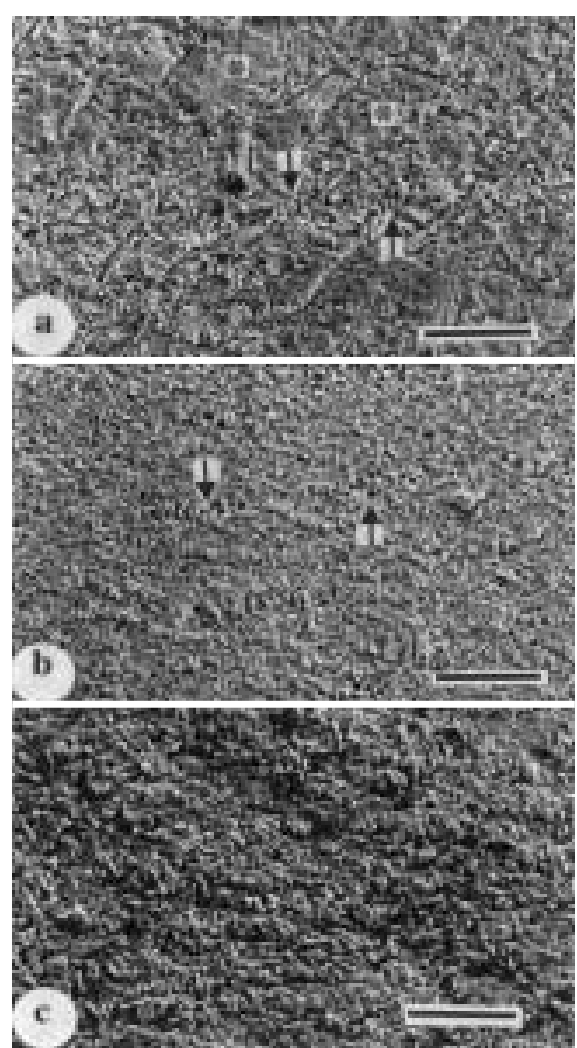

Fig. 7: Scanning electron micrographs of the etched palisade layer: a) the lower palisade layer. Note the irregular appearance of the crystallites in this region $\left({ }^{*}\right)$ and the $1 \mu \mathrm{m}$ pores running in bands (arrows), which are also present in the inter-dendritic growth areas of the mammillary layer (scale bar = $10 \mu \mathrm{m})$; b) the crystallites have become smaller in the mid-palisade region and the pore banding is still visible (arrows) (scale bar $=10 \mu \mathrm{m})$; c) near the VCL a more amorphous appearance is found with only jagged structures visible (scale bar $=10 \mu \mathrm{m}$ ).

(Fig. 6c). The size of the crystallites varies between 90 and $140 \mu \mathrm{m}$ in diameter.

In the polished etched samples of the palisadelayer, thecrystallites appear to be themammillary layer. Themicropores are not as clearly distinguished in the palisade layer and appear to vary in number depending on their position in the layer, decreasing as the VCL is approached. This variation is associated with differencesin the delineated crystallitemorphology of thislayer (Fig. 7a-C). In the region closest to the dendritic mammillary layer, the delineated crystallites have an irregular appearance (Fig. 7a). The larger micropores run in horizonsimilar to the inter-dendritic regions of

tally-arranged bands across the etched surface in a similar manner to that found in the inter-dendritic regions. The smaller pores are observed in the material between the bands but with no clear pattern of distribution. Morphologically the delineated crystallites becomesmaller the closer towards the VCL theobservation is made. In the middle portion of the palisade layer the large and small pores are still present, with the horizontal arrangement of the former becoming clearer (Fig. 7b). In the area closest to the $V C L$, the crystallite structures become fused, with a few jagged areas of delineation (Fig. 7c). Thesmaller poresseen in the lower parts of this layer are visible but the larger, $1 \mu \mathrm{m}$ pores, are reduced in number. The closer the palisade layer approaches the VCL the more amorphous its appearance becomes.

The VCL, in the polished etched samples, has an amorphous appearance with few identifiable features (Fig. 8a). The layer is divided into blocks, thejunctional zones of which appear as spaces devoid of any crystalline or organic material (Fig. 8a). A clear demarcation between the blocks and the upper palisade layer is not always present and the block structure may appear to be continuous with the palisade layer. In the specimens that had been prepared using the traditional fracture method this layer was also observed to have an amorphous appearance, with no evidence of pores. However, when the fracture was in an oblique direction in relation to the outer surface of the egg, additional features became apparent. The upper portion of the VCL had a layered appearance where each layer was approximately $1.4 \mu \mathrm{m}$ thick, progressing in astepwisefashion towards the surface (Fig. 8b). The last layers had a fractured 'crumbly' appearanceand were surmounted by a thin laminar surface sheet (Fig. 8c).

\section{DISCUSSION}

The outer eggshell membrane and its relationship to the calcification process has been extensively studied in the chicken $^{2,8,12,21,26}$. In the chicken the OSM is composed of types I, V and X collagens, with calcification occurring around nucleation centres containing mucopoly-

Table 1: Mean percentage weight (mean \%) and standard deviation (SD) of the elements carbon, calcium and sulphur in the cup and fibrous portions of the OSM, as determined by EDS analysis.

\begin{tabular}{|c|c|c|c|c|c|c|}
\hline & \multicolumn{2}{|c|}{ Carbon } & \multicolumn{2}{|c|}{ Calcium } & \multicolumn{2}{|c|}{ Sulphur } \\
\hline & Mean \% & SD & Mean \% & SD & Mean \% & SD \\
\hline Cup & 29.46 & 10.93 & 31.58 & 11.36 & 2.32 & 1.61 \\
\hline Membrane & 59.95 & 1.87 & 9.44 & 2.66 & 5.21 & 0.45 \\
\hline
\end{tabular}

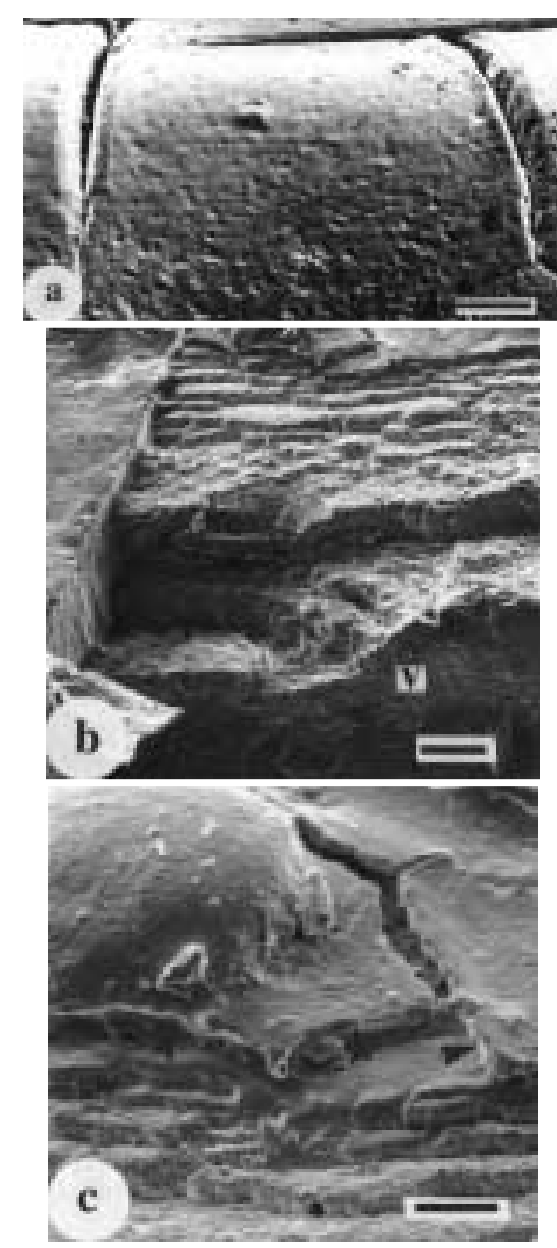

Fig. 8: Scanning electron micrographs of the etched and fractured VCL: a) etched specimen showing the block formation of the VCL and its amorphous appearance (scale bar = $10 \mu \mathrm{m}$ ); b) an obliquely fractured specimen, showing the multiple layers and 'crumbly' appearance of the upper VCL. Note the amorphous appearance of the vertical fracture face $(\mathrm{v})$, which is similar to the etched specimen (scale bar = $10 \mu \mathrm{m})$; c) upper few layers of the VCL with the thin laminar layer on the outermost surface (arrowheads) (scale bar $=10 \mu \mathrm{m})$.

saccharides and keratin sulphate ${ }^{3,10,21}$. The initiation and process of calcification in the domestic hen was determined at the ultrastructural level when the organic cores of the mammillary knobs were first described $^{12}$. In the present study no cores, as described for the chicken, were found on the OSM of the ostrich. This suggests that the usual comparison of the ostrich to the chicken, in terms of egg structure, may be erroneous. Whereas the ostrich eggshell shows certain structural characteristics similar to other species, for example the cup structures on the OSM resemble similar structures found associated with the OSM of the passion bird (A gapornis rosei collis) ${ }^{5}$, it differs markedly from that of the chicken. In the ostrich, the cup-shaped structures on the outer surface of the OSM appear to be composed of the same or similar material to the underlying OSM, but have a higher 
degree of calcification compared to the fibres of the membrane. It has been suggested that type $X$ collagen acts as a barrier and inhibitory compound in order to establish the boundaries of calcification ${ }^{4}$. If the cup is composed of type $X$ collagen, then calcification is probably initially confined within thebowl of thesestructures.

The dome-shaped formations observed on the inner aspect of the shell, following the removal of the OSM, are consistent with the observations of Tyler and Simkiss ${ }^{28}$. These structures have a much more open formation compared to the base of themammillary layer in radial section. As these structures are removed by deep-etching, it is likely that they are the contents of the cup structures rather than nucleation points of the true calcified shell. Analysis of the cup's contents may help elucidate the initial crystallisation conditionsthat arepresent in thecup. The step-like configuration of the plate/rod crystall ites suggests that crystall isation in the cups is the result of successive waves of fluid being released into thecups. Crystallite growth, following each wave, may be halted by the presence of the cup wall, thus creating the domed structures that are visible after the removal of the OSM . The results of X-ray and electron diffraction studies on a similar area in the chicken, the mammillary knobs, are often in disagreement with regard to the orientation of the crystallites ${ }^{7,18}$. This may be explained by the present results, in that the diffraction studies undertaken would have examined only a small area of crystallite growth, whereas in this SEM study a moregeneral morphological picturehas been ascertained. Theopen configuration of these crystallite structures may be indicative of a calcium store that is used during embryonic development ${ }^{3}$, and could therefore correspond to the primary spherite as described by Mikhailov in un-incubated eggs ${ }^{16}$. The cup and its contents would correspond to the 'core' of the mammillary layer of the egg shell of the domestic hen. However, the appellation of 'core' to this structure would not be appropriate, as no penetration of the mammillary layer by these structures occurs as is the case in the domestic hen.

Theremoval of the domed structureson the inner aspect of the eggshell by deepetching reveals a crystallite morphology consistent with naturally occurring calcite crystals $s^{22}$. The size of the delineated crystallites are similar to the crosssectional measurements of the base of the dendritic columns of the mammillary layer. It is thus likely that the deepetching of this area has revealed the true basal surface of themammillary layer. The different grains within each crystall iteare consistent with crystallite growth in the presence of foreign substances ${ }^{29}$. The etched and polished specimens confirmed the presence of multiple crystallites that have a dendritic growth pattern with initial nudeation occurring on the surface of the OSM cups' contents. Such a growth pattern is consistent with fast growth under favourable conditions of temperature, ionic concentration, impurity levels and the local movements within thefluid that arepresent astheegg is passed through the uterus ${ }^{29}$. The presence of multiple dendritic structures is suggestive of multiple nucleation sites, probably situated on the surface of the contents of the OSM cups. These nucleation sites may be low-energy crystal faces, which promote crystal growth ${ }^{29}$. As dendritic crystallites grow, the calciumrich fluid will tend to pool in the areas between the growing dendritic crystallite columns. In such areas, growth will be slower, with crystallites forming in an increasingly random pattern, with no preferred orientation, as a result of local depletion of the calcium and limited access to thecal cium-rich solution ${ }^{18}$. If this is the case, a line of trapped air bubbles may be expected, when there is a disturbance to the 'pool', as is seen in these egg shells. As this slower growth builds up, it will limit access of the growing dendritic crystallitesto themedium, thushindering their growth. The slower growth conditions may also allow time for air-bubble formation, thus giving rise to the micropores seen in the inter-dendritic and palisade growth regions. It would be expected that the dendritic growth structures in themammillary layer would have a preferred orientation, as suggested by Cain and $\mathrm{Heyn}^{7}$, even though Perrot et al. could not find such an orientation for this layer in their study of the eggshell of the domestic hen ${ }^{18}$.

In the ostrich eggshell it is probablethat, as the calcification front proceeds, the intervening areas calcify and merge, thus limiting access to the calcium-rich fluid to only a few crystallite faces, in a similar manner to that observed for the domestic hen ${ }^{18}$. As the growth process becomes slower a morelateral growth phaseoccurs in the palisade layer and VCL. As no obvious columnar structures were found in the palisade layer of the ostrich eggshell in this study, contrary to that reported by Sparks and Deeming ${ }^{23}$, it is probable that the crystallites of this layer have no preferred orientation. This is contrary to findings for the crystallites that made up the calcite columns of the palisade layer of the chicken $\mathrm{egg}^{18}$. The calcification of theostrich egg at thispoint may bevia a series of growth spurts as the calcium-rich fluid is excreted from the cells lining the isthmus and uterus. As each successive wave of excretion takes place, it creates a small amount of turbulence, thus creating the small air bubbles seen in the palisade layer. As the calcification front proceeds, these air bubbles are engulfed and remain trapped within the growing shell. The slightly larger air bubbles may be formed as the following wave of solution is released from the glands. Thecloser to thefinished product, the less room there is for turbulence to occur, thus leading to a reduction in the number of air bubblesnearer theVCL and in the VCL itself.

The VCL has been variously described as being amorphous ${ }^{19}$ or having a fractured crystallineappearance ${ }^{24}$. This study suggests that both descriptions may be applicable. In both the etched and fractured samples, when theplane of fracture was vertical in respect to the shell's surface, the description of Richards et al. ${ }^{19}$ was observed. However, a more layered and fractured appearance was observed when this layer was fractured at an oblique angle relative to the shell's surface, thus corresponding to the description offered by Sparks and Deeming ${ }^{24}$. The layered appearance of the upper portion of the VCL is consistent with the findings of Perrot et al., who reported a preferred crystal orientation in the VCL of the domestic hen ${ }^{18}$. The oblique fracturealso revealsathin laminar layer (TLL) on the upper surface of the shell, which covers the underlying stepped layers, forming the upper portion of the VCL. The EDS profiles ${ }^{19}$ of the TLL and the stepped layers differ from those of the lower portion of the VCL. Richards et al. ${ }^{19}$ suggested that this layer may have an organic component to its composition, as it has a higher sulphur and carbon content in comparison to the lower portion of the $\mathrm{VCL}^{19}$. There is no additional organic layer covering the surface of the eggshell of this species, which is consistent with the reported structurefor theostrich eggshell ${ }^{24}$. The TLL and thoselayers immediately below may have originally been an organic cuticle, which, as a result of an evolutionary trend, has become incorporated within the VCL through a calcification process. Alternatively, this high organic matrix in the upper VCL may be the terminal stage of the total organic matrix of the shell. Biomineralisation naturally takes place on a thin organic matrix, which, although clearly present in the chicken egg ${ }^{1}$, has not been clearly demonstrated in the ostrich eggshell ${ }^{23}$. Should such a matrix be present in the ostrich eggshell, the occurrence of crystal- 
lites in the inter-dendritic region and the palisade layer outwards, may be explained in terms of Garcia-Ruiz's induced morphology crystallites model ${ }^{15}$. Thus, in these regions, the crystallite growth is directed by the laying down of the organic matrix, which acts as a membrane in terms of the Garcia-Ruiz model and as such controls the calcification process ${ }^{15}$.

The detailed ultrastructural observations of the calcified portion of the ostrich egg shell presented in this study suggest mechanisms for the formation of the various layers in this species. Failure of the various crystallite growth patterns, as a result of dietary imbalances or pathological conditions, may lead to the formation of the varioustypes of abnormal eggs that have been described ${ }^{14}$. The basic ultrastructural descriptions of the calcified portions of the shell presented in this study paves the way for comparative studies of eggshells from farms that expe rience high hatchling trauma rates and which may contribute to determining a structural cause for the problem. Such work may help to develop dietary or other interventions to overcome these problems.

Further investigation of the grains and crystallites revealed by the etching and deep-etching procedures will permit the elucidation of theinitial nucleation conditions and the kinetics of crystallisation involved in the initial formation of the ostrich eggshell.

\section{ACKNOWLEDGEMENTS}

We acknowledge the help of Professor J Soley from the Department of Anatomy, Onderstepoort Veterinary Faculty, University of Pretoria, who supplied the shells used in this study and Mr A Botha, Microscopy and Microanalysis Unit, University of Pretoria, for his assistance with the EDS.

\section{REFERENCES}

1. Arias J L, Carrino D A, Fernàndez $M S$, Rodríguez J P, Dennis J E, Caplan A I 1992 Partial biochemical and immunocytochemical characterization of avian eggshell extracellular matrices. Archives of Biochemistry and Biophysics 298: 293-302

2. Arias J L, Fernàndez M S, Caplan A I 1991 Absencefrom avian eggshell membranes of epitopes recognized by anti-keratin antibodies. Poultry Science 70: 1647-1650

3. Arias J L, Fink D J, Xiao S-Q, Heuer A H, Caplan A I 1993Biomineralization and eggshells: cell-mediated acellular compartments of mineralized extracellular matrix. International Review of Cytology 145: 217-250

4. Arias J L, Nakamura $O$, Fernàndez $M S$, Wu J-J, KniggeP, EyreD R, Caplan A I 1997 Role of typeX collagen on experimental mineralization of eggshell membranes Connective Tissue Research 36: 21-33

5. Board R G 1982 Properties of avian egg shells and their adaptive value. Biological Reviews 57: 1-28

6. Bunk M J, Balloun SL 1978UItrastructureof the mammillary region of low puncture avian eggshells. Poultry Science 57: 639-647

7. Cain C J, Heyn A N J 1964 X-ray diffraction studies of the crystalline structure of the avian egg shell. Biophysical Journal 4: 23-39

8. Candlish J K 1969 The outer shell membrane of the avian egg shell as a reticular structure. British Poultry Science 11: 341-344

9. Carnarius K M, Conrad K M, Mast M G, Macneil J H 1996 Relationship of eggshell ultrastructure and shell strength to the soundness of shell eggs. Poultry Science 75 : 656-663

10. Carrino D A, Dennis J E, Wu T-M, Arias J L, Fernàndez $M S$, Rodriguez $J$, Fink $D J$, Heuer A H, Caplan A I 1996 The avian eggshell extracellular matrix as a model for biomineralization. Connective Tissue Research 35: 325-329

11. Christensen V L, Davis G S, Lucore L A 1996 Eggshell conductance and other functional qualities of ostrich eggs. Poultry Science 75: 1404-1410

12. Creger CR, Phillips H, ScottJ T 1976Formation of an eggshell. Poultry Science 55: 1717-1723

13. Deeming D C 1995 The ratite egg. In Drenowatz C (ed.) The ratite encyclopedia. Ratite Records Inc., Texas: 93-101

14. Deeming D C 1997 Ratite egg incubation. Ratite Conference, Oxford, 1997
15. Garcia-Ruiz M 1985 On the formation of induced morphology crystal aggregates. Journal of Crystal Growth 73: 251-262

16. Mikhailov K E 1980 The principle structure of the avian egg-shell: data of SEM studies. A cta Z oologica. Cracoviensia 30: 53-70

17. Parsons A H 1980 Structure of the eggshell. Poultry Science 61: 2013-2021

18 Perrot H R, Scott V D, Board R G 1981 Crystal orientation in the shell of the domestic fowl: an electron diffraction study. Calcified Tissue International 33: 119-124

19. RichardsP D G, BothaA J, Richards P A 1998 Vertical crystal layer of ostrich eggshell: one or two layers? Proceedings of the M icroscopy Society of Southern Africa 27: 134

20. Richards P D G, Lee M E, Richards P A 1997 Determination of avian eggshell ultrastructure - methodology. Proceedings of the M icroscopy Society of Southern A frica 27: 134

21. Robinson D S, King N R 1968 Mucopolysaccharides of an avian egg shell membrane. Journal of the Royal Microscopical Society 88: 13-22

22. Rogers A F, Reed R D 1926Sand-calcitecrystals from Monterey County, California. A merican M ineralologist 11: 23-28

23. Sparks N H C, Deeming D C 1996 Ostrich eggshell ultrastructure- astudy using electron microscopy and x-ray diffraction. In Deeming D C (ed.) Improving our understanding of ratites in a farming environment, Proceedings Ratite Conference, Oxford: 164-165

24. Sparks N 1995 Shell structure and hatchability. International $\mathrm{H}$ atchery Practice 10: 21

25. Sauer E G F 1972 Ratite eggshells and phylogenetic questions. Bonner Zoologische Beiträge 1: 3-48

26. Sauer E G F, Sauer E M, Gebhardt M 1975 Normal and abnormal patterns of struthious eggshells from South West Africa. Biomineralisation 8: 32-54

27. Stemberger B H, Mueller W J, Leach R M 1977 Microscopic study of the initial stages of egg shell calcification. Poultry Science 56: 537-543

28 Tyler C, Simkiss K 1959 A study of the egg shells of ratite birds. Proceedings of the Zoological Society, London 133: 201-243

29. Van Hook A 1961 Crystallization. Theory and practice. Chapman Hall, London: 92157

30. Van Toledo B, Parsons A H, Coombs G F 1982 Role of ultrastructure in determining eggshell strength. Poultry Science 61: 569-572 